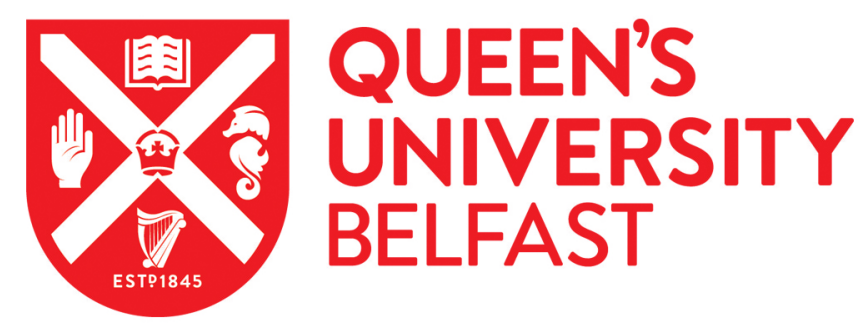

\title{
Integrating Behavioral Science with Mobile (mHealth) Technology to Optimize Health Behaviour Change Interventions
}

Walsh, J. C., \& Groarke, J. M. (2019). Integrating Behavioral Science with Mobile (mHealth) Technology to Optimize Health Behaviour Change Interventions. European Psychologist, 24(1), 38-48.

https://doi.org/10.1027/1016-9040/a000351

Published in:

European Psychologist

Document Version:

Peer reviewed version

Queen's University Belfast - Research Portal:

Link to publication record in Queen's University Belfast Research Portal

Publisher rights

(c) 2018 Hogrefe Publishing. This work is made available online in accordance with the publisher's policies. Please refer to any applicable terms of use of the publisher.

\section{General rights}

Copyright for the publications made accessible via the Queen's University Belfast Research Portal is retained by the author(s) and / or other copyright owners and it is a condition of accessing these publications that users recognise and abide by the legal requirements associated with these rights.

Take down policy

The Research Portal is Queen's institutional repository that provides access to Queen's research output. Every effort has been made to ensure that content in the Research Portal does not infringe any person's rights, or applicable UK laws. If you discover content in the Research Portal that you believe breaches copyright or violates any law, please contact openaccess@qub.ac.uk. 


\title{
Integrating Behavioural Science with Mobile (mHealth) Technology to Optimise Health Behaviour Change Interventions.
}

\begin{abstract}
The feasibility and potential for mobile technology to effect change in health-related outcomes for chronic disease is enormous. According to the European Commission (2014) among the world's population of 7 billion, there are over 5 billion mobile devices and over $90 \%$ of the users have their mobile device near them 24 hours a day. This provides a huge opportunity for behaviour change, and one that health psychologists have already begun to address. However, research evidence to date is limited and research is falling behind the rapid pace of new technology developments. Much of the research published to date reports low effect sizes and lack of sustained usage of mHealth technology over time which highlights the need to further understand how engagement with, and effectiveness of mHealth interventions can be optimised. The European Commission carried out a public consultation in 2016 on the issues surrounding the use of health and wellbeing apps and found that a lack of global standards were a significant barrier to their effective use. Recently, the WHO mHealth Technical Evidence Review Group developed the mHealth evidence reporting and assessment (mERA) checklist to specify the evidence-based content of mHealth interventions. Health psychologists play a key role in the development of mHealth solutions to foster behaviour change, particularly in the management of chronic disease. This article discusses the current challenges facing a widespread integration of mobile technology solutions into self-management of chronic disease including issues around security and regulation, as well as investigating potential solutions to overcoming these barriers.
\end{abstract}

Key Words: mHealth, Health Behaviour Change, Digital health, Behaviour change techniques 
This narrative review paper will open by identifying some current challenges in healthcare before outlining the role of behavioural science in addressing these challenges. The potential for new mobile technologies to facilitate health behaviour change will be evaluated, and the evidence base for existing mobile health (mHealth) interventions will be reviewed. The potential for mHealth to increase our understanding of behaviour change is disscussed. Next the paper will present the World Health Organisation's recommendations for conducting high quality research on the efficacy of mHealth interventions, and will describe the European Commissions guidelines for increasing the user-acceptability and uptake of mHealth apps. Finally, future directions for the development of technologies and interventions that incorporate the principles of behavioural science will be suggested.

\section{Modern Challenges for Health and Wellbeing: Self-Management of Healthy Lifestyle Behaviour}

During the $20^{\text {th }}$ century the leading causes of death have changed from infectious diseases to those that relate to unhealthy behaviour and lifestyle. The World Health Organization (WHO) estimates that around $63 \%$ of deaths globally are a result of lifestyle related diseases and further estimates that by 2020 , tobacco will account for $10 \%$ of all deaths worldwide (Alwan, 2011). Physical inactivity increases all-cause mortality risk by $20-30 \%$, excessive alcohol use accounts for about $3.8 \%$ of deaths worldwide, and an unhealthy diet is linked to heart disease, stroke, diabetes, and cancer. Many of the leading causes of death in Europe (i.e., Ischaemic Heart Disease, Cerebrovascular Disease, Cancer, and Chronic Respiratory Disease) are all strongly related to behaviour (Office for National Statistics, London, 2010). In 2016 the American Heart Association report stated that approximately $80 \%$ of cardiovascular diseases (CVDs) can be prevented through not smoking, eating a healthy diet, engaging in physical activity (PA), maintaining a healthy weight, and controlling high blood pressure, type 2 diabetes mellitus, and elevated lipid levels (Mozaffarian et al., 2016). A 10\% weight reduction in men aged 35 to 55 through dietary modifications and exercise would produce an estimated $20 \%$ decrease in coronary artery disease, it would also lower the degree of degenerative arthritis, gastrointestinal cancer, diabetes, stroke, and heart attack.

Considering the significant role of lifestyle behaviours in the development of chronic health conditions, the promotion of good health should move from a biomedical model focused on 
the physical and biological roots of illness, towards a focus on the management of behaviours that support or diminish health. Healthcare costs have been rapidly increasing in part because the diseases that are currently most prevalent are chronic in nature. Chronic diseases require continual treatment and monitoring and are thus more costly. Successful modification of health behaviours may help to reduce both the numbers of deaths and the incidence of preventable disease, as well as, make a dent in the more than billions of euro spent yearly on health and illness internationally (Mozaffarian et al., 2016). Therefore, encouraging people to adopt healthier lifestyles, and supporting those who wish to do so, is a highly desirable goal.

\section{Health Behaviour Change}

In view of the evidence of the link between behaviour and health, it is clear that the implementation of evidence-based practice and public health depends on behaviour change. Changing unhealthy lifestyle behaviours, namely poor nutrition and lack of PA, are the cornerstone to preventing chronic disease (CDC; Tuso, 2014). Thus, behaviour change interventions are fundamental to the effective practice of public health and clinical medicine. 'Behaviour change interventions' can be defined as coordinated sets of activities designed to change specified behaviour patterns (Michie et al., 2011). Interventions can be used to increase both uptake and optimal use of effective clinical services (e.g., vaccination, screening) and to promote healthy lifestyles (e.g., increase physical activity, quit smoking).

Behaviour change theories can be used to predict outcomes and aid the development of interventions that target healthy and unhealthy behaviours. There are many long-standing, influential theories, including the Theory of Planned Behaviour (Ajzen, 1985), Goal-Setting Theory (Locke \& Latham, 1990), the Health Belief Model (Janz \& Becker, 1984), and Bandura's (1986) Self-Efficacy Theory. For instance, a review of studies on smoking cessation, weight management, physical activity, and alcohol abuse, found that interventions can increase self-efficacy and this increase is related to subsequent health behaviour change (Strecher, Mc Evoy DeVellis, Becker \& Rosenstock, 1986). Another review of behaviour change interventions found that greater use of theory was associated with increased effect sizes, and that interventions based on the Theory of Planned Behaviour had particularly strong effects on health behaviour change (Webb, Joseph, Yardley, \& Michie, 2010). 
However, critiques have also highlighted the limitations of this theory (Sniehotta, Presseau, Araújo-Soares, 2014).

A single theory may not be sufficient to explain the complexity of human behaviour, and perhaps a more over-arching approach to behaviour change theory is required. One such approach is the Behaviour Change Wheel (Michie et al., 2011) developed by synthesising 19 different frameworks of behaviour change. In the process of designing a behaviour change intervention The Behaviour Change Wheel provides a useful way of linking a model of behaviour to common functions of interventions to change that behaviour (e.g., education, persuasion, coercion, incentivisation), and in turn, linking these intervention functions to policy categories (e.g., service provision, guidelines) that facilitate behaviour change. It is hoped that taking a more systematic approach to intervention development will lead to more effective interventions.

A related approach is the Behaviour Change Technique Taxonomy (version 1) (Michie et al., 2013). Behaviour Change Techniques (BCTs) are defined as the observable, replicable components of behaviour change interventions. The techniques making up the taxonomy have been synthesised from related constructs drawn from theories and frameworks across clinical and health psychology research and pracitce. Using the BCT taxonomy to design effective interventions is therefore not inconsistent with other theoretical approaches. The BCT taxonomy contains 93 BCTs, such as, 'goal-setting', 'feedback', 'self-monitoring', and 'social support' A recent review found that the use of relevant BCTs significantly increased the success of weight loss programmes (Hartmann-Boyce, Johns, Jebbs, \& Aveyard,, 2014). Further, a systematic review of 85 online interventions for health behaviour change found that interventions with a greater number of BCTs had larger effects than interventions with fewer BCTs (Webb et al., 2010).

There are a number of stages to developing behaviour change interventions, specifically; planning and design, early development, acceptibility and feasability testing, and evaluation. Identifying the active components of interventions using the BCT taxonomy has become an increasingly common way of developing the theoretical content of interventions designed to 
change behaviour at the planning and design phase of intervention development. The early development phase can also benefit from selecting appropriate BCTs and determining effective modes of delivery (e.g., face to face, text message, app, virtual reality).

Acceptibility and feasability testing with stakeholders and users can identify if the selection and delivery of BCTs is suitable for the population and desired outcome. Finally, if fractional factorial design (Box \& Hunter, 1961) studies are employed they can also evaluate which BCTs are effective for the target group, or standard RCTs can determine the efficacy of intervention for behaviour change.

Statistical tests like meta-analyses and meta-regressions have been used to identify the most effective BCTs for changing different behaviours in different populations. For instance, the BCTs of 'goal-setting' and 'self-monitoring' of behaviour are recommended for increasing PA and healthy eating in obese adults (Samdal, Elde, Barth, Williams, \& Meland, 2017). In contrast, the most effective BCTs targeting diet and PA in type 2 diabetes were found to be 'action planning', 'behavioural practice', 'instruction of behaviour', and 'demonstration of behaviour' (Cradock, Ó’Laighin, Finucane, Gainforth, Quinlan \& Martin Ginis, 2017). Appropriate BCTs can be selected based on a review of previous research, and will aid developers to create more effective behaviour change interventions. (both traditional and digital).

\section{Mobile Technology and Health (mHealth)}

Mobile health technologies (mHealth) have the potential to improve access to and use of health services, particularly among high-need and high-cost populations that have not been effectively engaged with in public health research and practise to date (Singh et al., 2016). mHealth is defined as the practise of medicine, public health, and allied healthcare or selfcare supported by mobile devices (e.g., smartphones, tablet computers, and wearable activity monitors). The aim of mHealth is to capitalise on the rapid uptake of information and communication technologies (ICT) to improve health system efficiency and health outcomes. The past decade has witnessed the rapid proliferation of mHealth solutions throughout developed and developing countries. 
According to the European Commission (2014) among the world's population of 7 billion, there are over 5 billion mobile devices and over $90 \%$ of the users have their mobile device near them 24 hours a day. Mobile phone use has almost achieved complete penetration with $96 \%$ of the global adult population having a mobile phone subscription (Sanou, 2015). Internet access is rapidly growing with approximately 400 million internet users globally in 2000 , rising to 3.2 billion by 2015 . This provides a huge opportunity for behaviour change, and one that health psychologists have already begun to address.

There are an estimated 97,000 mHealth apps on the market. More than two thirds of the apps cater for the consumer fitness and wellness sector, and one third are targeting health professionals and aiming to increase the efficiency of healthcare systems (Research2Guidance, 2013). At the same time, there are a number of barriers to effective self-management of chronic conditions using mobile technologies. For one, access to reliable internet and quality devices is not distributed equally within and between nation states. Another outstanding issue with mHealth apps and interventions is how best to sustain user engagement and overcome the declining rate of usage observed over time (Tatara, Arsand, Skrovseth \& Hartvigsen, 2013; Kohl, Crutzen \& de Vries, 2013).

\section{mHealth and interventions for health behaviour change.}

One way the advancement of technology offers simple, convenient approaches to facilitate self-management of health is by enabling easy monitoring of diet and physical activity (Thomas \& Bond, 2014). For example, two recent randomised controlled trials found that a simple smartphone pedomoter significantly increased physical activity (Glynn et al., 2014; Walsh, Corbett, Hogan, Duggan, \& McNamara, 2016). In the study by Glynn et al., (2014) 90 participants recruited from a primary care setting received information about the benefits of exercise. After eight weeks daily step count had significantly increased for all participants. However, participants in the intervention condition used a pedometer app and had a significantly greater increase in steps per day relative to the control group receiving information only. A technological advance on pedometer apps are the wearable activity monitors, such as the commercially available accelerometer bracelets and smart watches (i.e., Fitbit, Jawbone, Garmin etc.). In a study with overweight/obese adults these wearable activity monitors were associated with increased step count at 6 week follow-up. Interestingly, participants' level of engagement with the mobile app accompanying the wearable device 
(operationalised as the number of user logins) was associated with increased steps (Wang et al., 2016).

Pedometer apps and activity monitors provide visual feedback on a user's number of steps and physial activity level. This feature of the technology can potentially deliver and enable the BCTs of 'self-monitoring', 'goal-setting' and 'action planning'. Two studies using pedometer apps found that physical activity increases were greater in experimental conditions where pedometer use was combined with the BCT of 'goal-setting' in relation to increasing daily step count (Compernolle, Cardon, De Bourdeaudhuij, De Cocker \& Vandelanotte, 2015; Mansi, Baxter, Hendrick, Higgs, Milosavljevic and Tumilty; 2015). De Vries, De Groot, Kooiman, Van Brussel and Van Ittersum (2016) conducted a systematic review and meta-analysis on the efficacy of activity monitors to increase physical activity in an obese population finding that physical activity gains were significantly higher when the BCTs 'goal-setting' and 'feedback' were incorporated into the design of mHealth behaviour change interventions. Conroy, Yang and Maher (2014) reviewed the BCTs present in 167 highlyranked mobile apps for PA. The most common BCTs in these popular apps were 'instruction on how to perform behaviour', 'modelling behaviour', 'feedback', and 'goal-setting'. However, the number of BCTs present in each app was limited (typically less than four). The authors caution that users seeking to increase PA may therefore need to use more than one app to effectively change their behaviour.

The use of mHealth may also encourage adherence to a treatment plan (Doughty, 2011). There is evidence emerging from a systematic review of 13 studies that electronic reminder devices and short message service (SMS) reminders increase patients' adherence to chronic medication, at least in the short term (up to 6 month follow-up) (Vervloet, Linn, van Weert, de Bakker, Bouvy \& van Dijk, 2012). A larger review of 107 articles was less conclusive reporting that mHealth solutions aimed at adherence have the potential to improve chronic disease management, but that the evidence for its efficacy is mixed. However, more than half (56\%) of 27 RCTs reviewed did find significant improvement in adherence behaviour in patients with chronic diseases, with the majority of interventions using SMS reminders (Hamine, Gerth-Guyette, Faulx, Green \& Ginsburg, 2015). Another review of 20 mHealth interventions for increasing adherence to medication reported that despite significant heterogeneity in the design and quality of interventions, $65 \%$ of studies reported positive effects on adherence (Anglada-Martinex, Riu-Viladoms, Martin-Conde, Rovira-Illamola, Sotoca-Momblona, \& Condina-Jane, 2014). 
The results of a good number of existing reviews in different populations have demonstrated the potential for mHealth to increase adherence, yet the evidence is currently incolclusive. Non-adherence behaviour is complex and therefore complex interventions are required for it to change. Qualititative comparative analysis of a systematic review of 60 complex interventions was used to identify combinations of BCTs that were most effective for improving medication adherence in outpatients with chronic conditions. Improvement in adherence was reported in more than half of the studies (57\%). Of these studies, there were seven different configurations of BCTs that increased adherence. However, the most common and efficacious combination of techniques was 'increasing knowledge' coupled with ‘increasing self-efficacy’ (Kahwati, Viswanathan, Golin, Kane, Lewis, \& Jacobs, 2016). There are a wide range of apps available for increasing medication adherence. A content analysis of the BCTs present in 166 such apps reported that 12 of a possible 96 BCTs were present across these apps, and that $96 \%$ of the apps included the BCTs of 'action-planning', and 'prompting/cues'. More than one third of the apps that were reviewed featured the BCTs 'self-monitoring' and 'feedback on behaviour' (Morrissey, Corbett, Walsh \& Molloy, 2016). It is noteworthy that none of the available apps included the BCTs that were found to be most effective for increasing adherence in the qualitative synthesis by Kahwati et al. (i.e., 'increasing knowledge' or 'increasing self-efficacy'), indicating there is opportunity for greater integration of behavioural science theory and research in the design of apps, leading to improved quality and efficacy of mHealth interventions for improving adherence behaviour.

The current mHealth evidence is disseminated in multiple forms including peer reviewed literature, white papers, reports, presentations, and blogs. The evidence base is heterogenous in quality, completeness, and objectivity of the reporting of mHealth interventions - thus making comparisons across interventions difficult. Despite the emergence of hundreds of mHealth studies and initiatives, there remains a lack of rigorous, high quality evidence on the efficacy and effectiveness of such interventions (Agarwal et al., 2015). To date, few developers of digital health interventions have specified how characteristics of their intervention map onto underlying evidence-based theories and techniques (Morrissey et al., 2016) and unsurprisingly, the current evidence around the effectiveness of technological devices as a health behaviour change tool is limited. Some researchers have had success in the field. As described in the previous section, Glynn et al. (2014) had success in increasing physical activity in a primary care population using a pedometer app. However, this study 
along with others requires clearer specification of the behaviour change techniques (BCTs) employed along with the underlying theory-based mechanisms of behaviour change. There are many studies and systematic reviews signalling the promise of mHealth interventions for health behaviour change. However, the reporting of complex behavioural health interventions, digital and traditional, often lacks sufficient details to know exactly what scientific basis was used to develop the intervention and how the intervention was offered to participants.

\section{The Contribution of mHealth to Research and Theory on Health Behaviour Change}

Thus far, we have focused on the benefit of incorporating the principles of behavioural science into the design of mHealth interventions. Yet, it is also true that the advent of mHealth can benefit behavioural science research and theory. Traditionally, assessments of health behaviour have relied heavily on self-report (e.g., self-reported smoking, food diaries, medication adherence etc.), which are beset with problems of poor recall, inaccuracies, and socially desirable responding. In contrast, mobile technology provides opportunity for objective measurement of both health behaviour and health outcomes. For example, new wearable sensor technologies (e.g., bracelets, smart glasses) automatically provide minuteby-minute monitoring of objective measurements of behaviour (e.g., physical activity). This provides researchers with an excellent opportunity to measure health-related outcomes that were previously reliant on self-report or were confined to assessments in a clinical or lab environment (e.g., stress, mood, heart rate etc.). At the same time, the self-report measures coupled with objective clinical measures of health (e.g., BMI, cholesterol, blood pressure etc.) employed up to this point have helped to profile the links between health behaviours and health outcomes. Typically, self-report data is collected at a few distinct points in the trajectory of an illness (i.e., diagnosis, treatment, survivorship) or protocol of a research study (i.e., baseline, 6 and 12 months later), and whilst providing a useful snapshot of crosssectional data, they often failed to grasp the personal, contextual and social factors that influence self-management of health.

Further to this, the collection of psychological, social, and contextual variables that are passively recorded or tracked along with objective health data (e.g., GPS location, social media activity etc.) can be used to understand processes and outcomes of behavioural health 
interventions and for empirically testing behavioural theories in different contexts and settings (Hekler et al., 2013).

One of the problems with 'electronic health' (eHealth) and mHealth technology is that the speed of growth has not allowed enough time for theory development and research on its potential for health behaviour change. The growth in the number of eHealth as well as mHealth interventions in the last few years, and recent advances in technology have provided researchers with a golden opportunity for empirically testing behavioural theories in "realworld" contexts within health service delivery models. Digital platforms allow for greater specification of existing behavioural theories and models. Methodologies derived from mHealth could help define how constructs relate to one another over time, and the predicted magnitude and direction of those relations (Hekler et al., 2016b). For example, mHealth research could determine the temporal and dynamic relationships between effective components of an intervention, such as BCTs (e.g., 'goal-setting' and 'feedback'), psychological constructs (e.g., self-efficacy), and behavioural outcomes (e.g., physical activity). These potential interdependencies between intervention functions and outcomes are currently not well understood.

As previously described, BCTs are associated with increased behaviour change (de Vries et al., 2016; Kahwati et al., 2016; Webb et al., 2010), and perceived self-efficacy and goals also positively influence behaviour change (Bandura \& Locke, 2003). Wearable sensors provide 'feedback' to individuals on their level of physical activity, enabling 'self-monitoring' of behaviour and progress towards physical activity goals. Analysis of objective behavioural data (i.e., PA) collected in real-time from wearable devices can be combined with measures of an individual's sense of self-efficacy. Empirical tests can then determine if self-efficacy varies dynamically in connection with achievement of physical activity goals, and what impact this has on behaviour (i.e., level of PA). Failure to reach PA goals will be associated with less PA, but does 'self-monitoring' of failures in 'goal-setting' also reduce an individual's self-efficacy, which may in turn, negatively impact health behaviour change over time?

The shift from traditional to digital platforms presents researchers with an excellent opportunity to both develop and test theories using behaviour change techniques (Hekler et al., 2016b). Whilst this is a novel and exciting area of enquiry, there are few studies in this 
area, and many of the early studies have been heavily criticised for lacking a strong evidence base in both design and implementation.

\section{World Health Organisation Guidelines for Reporting mHealth Research}

In an attempt to move towards the establishment of global standards for mHealth research, the WHO mHealth Technical Evidence Review Group developed the mHealth evidence reporting and assessment (mERA) checklist (Agarwal et al., 2016). This checklist was designed to improve the quality of future research, to facilitate screening of emerging evidence, and identification of critical evidence gaps. These guidelines were developed with a view to improving the reporting of evidence that should support policy makers in making decisions around mHealth intervention selection. The guiding principle for the development of these criteria was to identify a minimum set of information needed to define (1) what the mHealth intervention is (content), (2) how and with whom it was implemented (technical and user features) and (3) where it is being implemented (context), to support replication of the intervention.

\section{(1) Content of mHealth interventions: theory-based design.}

The WHO recommend that the original source of any educational content is reported, along with the theoretical basis for new or adapted content.

It is clear that the potential public health impact of new mHealth technologies can only be realized to the extent that digital health interventions are effective. The process of developing an effective mHealth intervention will benefit from applying evidence-based theories and techniques, as it will inform design characteristics (e.g., behaviour change techniques) and indicate optimum conditions under which interventions and their specific characteristics will be most effective (Peters et al., 2015).

This is important for digital health interventions given that they often require considerable initial investments in development (e.g., app development is timely and costly). Morrison (2015) suggests that to achieve long term sustainability of digital interventions more research on the effective elements of interventions instead of effective mHealth interventions is 
required. She argues that the reach and use of these interventions need more scientific input to increase the public health impact of internet-delivered interventions.

Also highlighting the need to adopt theory-based design principles is a recent review paper that makes 16 evidence-based recommendations for the development of mental health apps (MHapps) (Bakker, Kazantzis, Rickwood \& Rickard, 2016). Seven of the recommendations advocate improving MHapp design features through application of research and theory on mental health, in particular cognitive behavioural therapy. Many recommendations are consistent with the effective BCTs for health behaviour change described in this paper. For example, psychoeducation (i.e., providing 'information' about mental health) is recommended, as well as, reporting of thoughts, feelings, and behaviour (i.e., 'selfmonitoring'). The application of theory provides a useful starting point to design technological interventions, the current paper recommends behavioural science theory due to its compelling evidence base. As stated, interventions including more BCTs have stronger effects on health behaviour change (Webb et al., 2010). Yet, studies have found that commercially available apps do not contain a large amount of BCTs (Morrissey et al., 2016; Conroy, Yang \& Maher 2014).

(2) Stakeholder involvement in user design. The WHO also highlights the importance of involving end-users in the initial design phase to properly inform elements critical to success such as individuals' and community characteristics as well as to determine effective modes of intervention delivery.

There has been increased recognition and emphasis placed on the importance of involving users and stakeholders from the outset in intervention design and implementation. One method of approaching this is to take the person-based approach, for example, Yardley et al.'s (2015) work in this area. This approach involves in-depth qualitative research conducted with the users before the digital intervention is developed. This data is used to develop "guiding principles" that state the key objectives of the intervention, and describe the key features of the intervention required to achieve each objective. They posit that qualitative research is crucial at all stages of intervention development and evaluation, including planning and design, early development, acceptability and feasibility testing, and evaluation in clinical trials and real-life settings. 
The person-based approach is highly compatible with the more in-depth approaches that have evolved within the disciplines of information systems and human computer interaction, such as human-centred and user-centred design. These approaches seek to understand the user's knowledge, skills, behaviour, motivations, cultural background, and organizational context, and they involve users iteratively throughout development. Traditionally, user-testing has focused on utility and engagement, aiming to increase user's enjoyment of and motivation to use technology (Kim, Kim, Wachter, 2013; O’Brien, 2010) The person-based approach, however, is rooted within the discipline of health psychology and focuses primarily on the behaviour change techniques the intervention is intended to deliver, and their desired implementation by the people using the intervention. Similar to user-testing methods aiming to increase the use of technology, the person-based approach aims to increase participants' engagement with an intervention such that the intended outcomes of the behaviour change intervention can be realised.

Glynn et al. (2015) conducted qualitative research to explore target users' perspectives in the development of an app for self-management of hypertension. Patients with hypertension stated that "one size fits all" interventions to enhance self-management of lifestyle behaviour are undesirable. Rather, patients prefer a personalised programme via an app enabling them to prioritise their own approach to self-management. Themes identified in the reports from patients in this study highlight that the source of the mHealth "prescription" was also an important factor and "trust" in the technology was highlighted as a key factor in relation to its potential effect on engagement with healthcare providers and motivation for engagement. The introduction of a new technology or platform for engagement requires concerted efforts to alleviate patient concerns and to create confidence in terms of quality and security. Further, patients' motivation to use mobile technology was influenced by the potential of technology to provide BCTs such as, 'information', 'feedback', 'reward' and 'reinforcement' systems which could embed new self-management habits. The potential for technology to facilitate a personalised flow of communication between patient and healthcare provider was recognised as important as was the ability of technology to facilitate tailored messaging and feedback for patients. The flexibility and inherent motivational ability of newer technologies seems to have the potential to improve the ability of patients to engage in sustained behaviour change. However evidence of long term engagement is still lacking in many studies as the majority of users tend to stop using apps after just a few weeks (Kohl, Crutzen \& de Vries, 2013). The Mobile Application Rating Scale (Stoyanov, Hides, Kavanagh, Zelenko, Tjondronegoro, \& 
Mani, 2015) is a tool for assessing the quality of apps by reseachers, professionals, and clinicians that can be used to aid the design and development of better quality mHealth apps. A user-version of the scale was recently developed (Stoyanov, Hides, Kavanagh, \& Wilson, 2016) offering a reliable and valid quantitiatve approach to stakeholder involvement in user design.

(3) Context of intervention delivery. The WHO requests that researchers state what context or setting the mHealth intervention is taking place, the appropriateness of the intervention to the context, and any potential for adaptation to other contexts.

The mHealth intervention may be designed for a specific context, setting, or group of users. However, one advantage of mobile interventions is they can be designed in such a way that they can be delivered in any context, when needed. The ability of mHealth to collect time and place specific data opens up the potential for researchers to implement user-friendly interventions that are context and time appropriate. For example, interventions that provide behavioural support (e.g., 'information' about health consequences of behaviour) at key times when a person is receptive to such support, or at key places where the person has the opportunity to change their behaviour (e.g. Moller et al., 2017; Naughton et al., 2016). The use of mobile apps also enables the specification of dynamic temporal relationships e.g., timescale, latency, and delay (Naughton et al., 2016; Spruijt-Metz et al., 2015). From a theoretical perspective, work on prospective memory suggests that people make effective use of cues that are appropriate for goal attainment, and that people readily execute an intended action in response to a specified cue when the action cue is encountered later during ongoing activity (Brandimonte, Einstein, \& McDaniel, 1996). The recent study by Naughton et al. (2016) has shown promising finding using these methods with respect to smoking cessation. Smoking behaviour is particularly prone to lapse and relapse during quit attempts as cravings are often triggered by cues from a smoker's immediate environment. Naughton et al. developed a context-aware smoking cessation app, Q Sense, which uses a smoking episodereporting system combined with location sensing and geofencing to tailor support content and trigger support delivery in real-time as needed. The development of this app was based on explanatory sequential mixed-methods design where data collected by the app informed semi-structured interviews. Although underreporting of smoking occurred, the findings suggested that geofence-triggered support was regarded positively by participants. These new technological developments pave the way for 'big data' to drive algorithmic solutions to 
provide optimum contextual cues for intervention delivery (Hekler, 2016a). This combined with a theory-informed and person-based content design, creates the possibility for a timely, context-appropriate, personalised, and highly effective intervention to be delivered to participants.

Through widespread adoption, it is hoped that the use of these guidelines will standardise the quality of mHealth evidence reporting, and indirectly improve the quality of mHealth evidence. In order to develop evidence-based, effective, and user-friendly mHealth interventions, researchers must move towards a more collaborative and multidisciplinary approach using these European standards as a framework.

The European Commission carried out a public consultation in 2016 to investigate the issues surrounding the use of mobile technology in the promotion of health and wellbeing. Contributions came from both individuals and organisations (e.g., pharmaceutical industry, telecommunications companies, health care providers, patients' associations). The green paper on mobile health reported that a lack of global standards and regulation were a significant barrier to the effective use of mHealth technologies. Primary concerns included issues around privacy and data security. Contributors described the need for standardisation in data management practices, for example the use of encryption and user authentication, noting that increased transparency would increase a user's trust in applications. Concerns regarding patient safety were also raised. There is currently no quality certification or regulation of mHealth apps, and this introduces the possibility that misinformation provided by an app might result in poor self-management decisions (e.g., taking medication incorrectly).

Following this enquiry, the EU published a Code of Conduct for the development of new mHealth applications. The code provides practical guidelines for developers around issues of consent, privacy, data storage, and security. The aim is to guide key stakeholders in the safe use of these new health apps, and to increase trust among the users of mHealth apps that process personal and health data (European Commission, 2016). In addition, an expert panel was assembled to develop guidelines on procedures to develop safe and effective mHealth solutions (Ruck et al., 2016). The guidelines state that health apps should be user-friendly, desirable, credible, effective, reliable, secure, and safe for the target groups - including a formal risk assessment. 


\section{Future directions: Moving towards greater personalisation}

The use of new digital technologies allows for increased personalisation of mobile health interventions based on an individual's unique profile, thus increasing the likelihood of successful health behaviour change. One reason to develop personalised interventions is that patients have identified personalised solutions as more desirable than generic apps for selfmanagement (Glynn et al., 2015). As such, personalisation may enhance the success of health behaviour change interventions or may encourage greater engagement with mHealth. In a mental health context, tailored computerised cognitive behavioural therapy interventions have been found to be more effective than standardised interventions (Johansson et al., 2012; Nordgren et al., 2014; Silfvernagel et al., 2012).

Personalised solutions, however, pose another challenge for researchers to develop and monitor interventions that may change over time by adapting to the user's changing requirements and altered goals. For example, a goal of 'couch to $5 \mathrm{k}$ ' may be the focus over an initial six or eight week period, but a new goal or shift in focus and emphasis may be required to sustain levels of physical activity once this goal has been achieved, or indeed, to support continued goal-pursuit in the face of failure to achieve physical activity targets. This is particularly important in developing health behaviour change interventions that foster maintenance and long term sustainability of the desired health behaviour (Almirall et al., 2014). These high-level adaptive changes are more achievable using new technologybased interventions by using live algorithmic analysis based on data collected by an app, both sensor-based and user-inputted. This type of analysis is complex and therefore requires a strong multidisciplinary approach with information technologists, medics, and health psychologists working closely together to make sense of and capitalise on the quantity and quality of data generated by new health technology.

\section{Conclusion}

Rapid changes in technology coupled with recent developments in behavioural science provide an excellent opportunity to deliver personalised evidence-driven behaviour change interventions. This paper has reviewed current evidence on the impact of mHealth for 
improving health behaviour, focusing on the opportunity for behavioural science to improve mHealth interventions.

The European Commission and the World Health Organisation have published guidelines to improve the development, implementation and evaluation of mHealth interventions in an effort to maximise the full potential of these innovative tools. Specfically, the European Commission has called for greater efficacy of mHealth apps, and the WHO has asked that a more theory-based approach to intervention development be adopted. The use of BCTs has been associated with increased efficacy of interventions (Webb et al., 2010; de Vries et al., 2016; Kahwati et al., 2016). Therefore, behavioural science theory can meet these demands. The European Commission has also asked for increased usability and utility of health apps. The potential for mHealth to deliver effective BCTs was highlighted by patients as a design feature that would increase their motivation to use an app for self-management (Glynn et al., 2015). Further, increased usability can also be achieved by adopting the person-based approach (Yardley et al., 2015), or user-centered approach to designing mHealth interventions for self-management of chronic conditions.

This paper has advocated the application of behavioural science theory in the design of digital and traditional interventions for health behaviour change. In developing effective interventions researchers should involve stakeholders and users at all stages of the development process, and should explicitly identify and systematically apply evidence-based behaviour change techniques (BCTs) in the intervention. Health psychologists should work closely in multidisciplinary teams to efficiently and effectively to develop optimum solutions for effective health behaviour change. 
References

Agarwal S., Perry H. B., Long L. A., \& Labrique A. B. (2015). Evidence on feasibility and effective use of mHealth strategies by frontline health workers in developing countries: a systematic review. Tropical Medical and International Health, 20, 100314. doi:10.1111/tmi.12525. 25881735

Agarwal, S., LeFevre, A. E., Lee, J., L’Engle, K., Mehl, G., \& Sinha, C. et al. (2016). Guidelines for reporting of health interventions using mobile phones: mobile health (mHealth) evidence reporting and assessment (mERA) checklist. BMJ: British Medical Journal, 352. https://doi.org/10.1136/bmj.i1174

Ajzen, I. (1985). From intentions to actions: A theory of planned behavior. In Action control (pp. 11-39). Springer Berlin Heidelberg.

Almirall, D., Nahum-Shani, I., Sherwood, N. E., \& Murphy, S. A. (2014). Introduction to SMART designs for the development of adaptive interventions: with application to weight loss research. Translational Behavioral Medicine, 4, 260-274. https://doi.org/10.1007/s13142-014-0265-0

Alwan, A. (2011). Status Report on Noncommunicable Diseases 2010. Geneva, Switzerland; World Health Organisation. Retrieved from http://whqlibdoc.who.int/publications/2011/9789240686458_eng.pdf

Anglada-Martinez, H., Riu-Viladoms, G., Martin-Conde, M., Rovira-Illamola, M., SotocaMomblona, J. M., \& Codina-Jane, C. (2015). Does mHealth increase adherence to medication? Results of a systematic review. International journal of clinical practice, 69(1), 9-32.

Line 844 Bakker, D., Kazantzis, N., Rickwood, D., \& Rickard, N. (2016). Mental health smartphone apps: review and evidence-based recommendations for future developments. JMIR Mental Health, 3(1), e7.Bandura, A. (1986). The explanatory and predictive scope of self-efficacy theory. Journal of social and clinical psychology, 4(3), 359-373.

Bandura, A., \& Locke, E. A. (2003). Negative self-efficacy and goal effects revisited. Journal of applied psychology, 88(1), 87.

Box, G. E., \& Hunter, J. S. (1961). The 2k-p fractional factorial designs. Technometrics, 3(3), 311-351.

Brandimonte, M., Einstein, G. O., \& McDaniel, M. A. (Eds.). (1996). Prospective memory: Theory and applications. Mahwah, NJ: Erlbaum. 
Compernolle, S., Cardon, G., De Bourdeaudhuij, I., De Cocker, K. \& Vandelanotte, C. (2015). Effectiveness of a web-based, computer-tailored, pedometer-based physical activity intervention for adults: a cluster randomized controlled trial. Journal of medical Internet research, 17(2), e38

Conroy, D. E., Yang, C. H., \& Maher, J. P. (2014). Behavior change techniques in top-ranked mobile apps for physical activity. American Journal of Preventive Medicine, 46, 649 652. https://doi.org/10.1016/j.amepre.2014.01.010

Conroy, D. E., Yang, C. H., \& Maher, J. P. (2014). Behavior change techniques in top-ranked mobile apps for physical activity. American journal of preventive medicine, 46(6), 649-652.

Cradock, K. A., ÓLaighin, G., Finucane, F. M., Gainforth, H. L., Quinlan, L. R., \& Ginis, K. A. M. (2017). Behaviour change techniques targeting both diet and physical activity in type 2 diabetes: A systematic review and meta-analysis. International Journal of Behavioral Nutrition and Physical Activity, 14(1), 18.

de Vries, H. J., Kooiman, T. J., van Ittersum, M. W., van Brussel, M., \& de Groot, M. (2016). Do activity monitors increase physical activity in adults with overweight or obesity? A systematic review and meta-analysis. Obesity, 24(10), 2078-2091. Doughty, K. (2011). SPAS (Smart Phone applications) - a new form of assistive technology. Journal of Assistive Technologies, 5(2), 88- 94. doi: $10.1108 / 17549451111149296$

European Commission. (2014). Green Paper on Mobile Health (mHealth). Retrieved from http://europa.eu/!ug89mT

European Commission. (2016). Code of Conduct on privacy for mobile health applications. Retrieved from http://europa.eu/!Fg74Xm

Glynn, L. G., Hayes, P. S., Casey, M., Glynn, F., Alvarez-Iglesias, A., Newell, J., ÓLaighin, G., Heaney, D., O’Donnell, M., \& Murphy, A., W. (2014). Effectiveness of a smartphone application to promote physical activity in primary care: the SMART MOVE randomised controlled trial. British Journal of General Practice, 64(624), 384 - 391. https://doi.org/10.3399/bjgp14X680461

Glynn, L., Casey, M., Walsh, J., Hayes, P. S., Harte, R. P., \& Heaney, D. (2015). Patients' views and experiences of technology based self-management tools for the treatment of hypertension in the community: A qualitative study. BMC Family Practice, 16(1) 119. https://doi.org/10.1186/s12875-015-0333-7 
Hamine, S., Gerth-Guyette, E., Faulx, D., Green, B. B., \& Ginsburg, A. S. (2015). Impact of mHealth chronic disease management on treatment adherence and patient outcomes: a systematic review. Journal of medical Internet research, 17(2).

Hartmann-Boyce, J., Johns, D. J., Jebb, A., \& Aveyard, P. (2014). Effect of behavioural techniques and delivery mode on effectiveness of weight management: systematic review, meta-analysis and meta-regression. Obesity Reviews, 15(7), 598-609. doi.org/10.1111/obr.12165

Hekler, E. B., Buman, M. P., Poothakandiyil, N., Rivera, D. E., Dzierzewski, J. M., Aiken Morgan, A., McCrae, C. S., Roberts, B. L., Marsiske, M., \& Giacobbi Jr, P. R. (2013). Exploring behavioral markers of long-term physical activity maintenance: a case study of system identification modeling within a behavioral intervention. Health Education \& Behavior, 40(1), 51-62. https://doi.org/10.1177/1090198113496787

Hekler, E. B., Klasnja, P., Riley, W. T., Buman, M. P., Huberty, J., Rivera, D. E., \& Martin, C. A. (2016a). Agile science: Creating useful products for behavior change in the real world. Translational Behavioral Medicine, 6(2), 317-328. https://doi.org/10.1007/s13142-016-0395-7

Hekler, E. B., Michie, S. F., Rivera, D. E., Collins, Jimison, H. B., Garnett, C., Parral, S., \& Spruijt-Metz, D. (2016b). Advancing models and theories for digital behavior change interventions. American Journal of Preventive Medicine, 51 (5), 825-832, https://doi.org/10.1016/j.amepre.2016.06.013

Janz, Nancy K.; Marshall H. Becker (1984). The Health Belief Model: A Decade Later. Health Education \& Behavior. 11 (1): 1-47. doi: $10.1177 / 109019818401100101$

Johansson, R., Sjöberg, E., Sjögren, M., Johnsson, E., Carlbring, P., Andersson, T., ... \& Andersson, G. (2012). Tailored vs. standardized internet-based cognitive behavior therapy for depression and comorbid symptoms: a randomized controlled trial. PloS one, 7(5), e36905.

Kahwati, L., Viswanathan, M., Golin, C. E., Kane, H., Lewis, M., \& Jacobs, S. (2016). Identifying configurations of behavior change techniques in effective medication adherence interventions: a qualitative comparative analysis. Systematic reviews, 5(1), 83.

Kim, Y. H., Kim, D. J., \& Wachter, K. (2013). A study of mobile user engagement (MoEN): Engagement motivations, perceived value, satisfaction, and continued engagement intention.Decision Support Systems, 56, 361-370. 
Kohl, L. F., Crutzen, R., \& de Vries, N. K. (2013). Online prevention aimed at lifestyle behaviors: A systematic review of reviews. Journal of Medical Internet Research, 15(7), e146. doi: 10.2196/jmir.2665

Locke, E. A., \& Latham, G. P. (1990). A theory of goal setting and task performance. Englewood Cliffs, NJ: Prentice Hall.

Mansi, S., Baxter, D. G., Hendrick, P., Higgs, C., Milosavljevic, S., \& Tumilty, S. (2015). Investigating the effect of a 3-month workplace-based pedometer-driven walking programme on health-related quality of life in meat processing workers: a feasibility study within a randomized controlled trial. BMC public health, 15(1), 410.

Michie, S., Atkins, L., \& West, R. (2014). The behaviour change wheel. A guide to designing interventions. 1st ed. Great Britain: Silverback Publishing.

Michie, S. \& West, R. (2016). A Guide to Development and Evaluation of Digital Behaviour Change Interventions in Healthcare. 1st ed. Great Britain: Silverback Publishing.

Michie, S., Richardson, M., Johnston, M., Abraham, C., Francis, J., Hardeman, W., Eccles, M. P., Cane, J., \& Wood, C. E. (2013). The behavior change technique taxonomy (v1) of 93 hierarchically clustered techniques: building an international consensus for the reporting of behavior change interventions. Annals of Behavioral Medicine 46(1), 81-95. https://doi.org/10.1007/s12160-013-9486-6

Michie, S., van Stralen, M. M., \& West, R. (2011). The behaviour change wheel: A new method for characterising and designing behaviour change interventions. Implementation Science, 6(42). https://doi.org/10.1186/1748-5908-6-42

Moller, A.C., Merchant, G., Conroy, D.E., West, R., Hekler, E., Kugler, K. C., \& Michie, S. (2017). Applying and advancing behaviour change theories and techniques in the context of a digital health revolution: proposals for more effectively realizing untapped potential. Journal of Behavioural Medicine 40(1), 85 - 98. https://doi.org/10.1007/s10865-016-9818-7

Morrison, L. G. (2015) Theory-based strategies for enhancing the impact and usage of digital health behaviour change interventions: a review. Digital Health, 1, 110. https://doi.org/10.1177/2055207615595335

Morrissey, E. C., Corbett, T. K., Walsh, J. C., \& Molloy, G. J. (2016). Behavior change techniques in apps for medication adherence: A content analysis. American Journal of Preventive Medicine, 50(5), 143 - 146. https://doi.org/10.1016/j.amepre.2015.09.034 
Mozaffarian, D., Benjamin, E. J., Go, A.S., Arnett, D. K., Blaha, M. J., Dai, S., ... \& Turner, M. B. (2016). American heart association statistics committee and stroke statistics subcommittee. heart disease and stroke statistics - 2016 update: A report from the american heart association. HHS Public Access, 129(3), 28 - 292. doi:

10.1161/01.cir.0000441139.02102.80

Naughton, F., Hopewell, S., Lathia, N., Schalbroeck, R., Brown, C., Mascolo, C., McEwen, A., \& Sutton, S. A. (2016). Context-sensing mobile phone app (q sense) for smoking cessation: A mixed-methods study. JMIR mHealth and uHealth, 4(3), 106. doi: 10.2196/mhealth.5787

Nordgren, L. B., Hedman, E., Etienne, J., Bodin, J., Kadowaki, Å., Eriksson, S., ... \& Carlbring, P. (2014). Effectiveness and cost-effectiveness of individually tailored Internet-delivered cognitive behavior therapy for anxiety disorders in a primary care population: A randomized controlled trial.Behaviour research and therapy, 59, 1-11.

O'Brien, H. L. (2010). The influence of hedonic and utilitarian motivations on user engagement: The case of online shopping experiences. Interacting with computers, 22(5), 344-352.

Office for National Statistics. (2010). Deaths Registered in England and Wales in 2010, by Cause. Office for National Statistics, London. Retrieved from https://www.ons.gov.uk/peoplepopulationandcommunity/birthsdeathsandmarriages/de aths

Peters, G. J. Y., de Bruin, M., \& Crutzen, R. (2015). Everything should be as simple as possible, but no simpler: towards a protocol for accumulating evidence regarding the active content of health behaviour change interventions. Health Psychology Review, 9, 1-14. doi:10.1080/17437199.2013.848409

Ruck, A., Bondorf, S. W., \& Lowe, C. (2016). EU guidelines on assessment of the reliability of mobile health applications. ( $2^{\text {nd }}$ Draft.). Retrieved from http://c.ymcdn.com/sites/echalliance.com/resource/resmgr/Docs/eHealth_week/Secon ddraftmHealthassessment.pdf

Sanou, B. (2015). ICT data and statistics division: Facts \& figures. Geneva, Switzerland: International Telecommunication Union (ITU). Retrieved from https://www.itu.int/en/ITUD/Statistics/Documents/facts/ICTFactsFigures2015.pdf

Silfvernagel, K., Carlbring, P., Kabo, J., Edström, S., Eriksson, J., Månson, L., \& Andersson, G. (2012). Individually tailored internet-based treatment for young adults and adults 
with panic attacks: randomized controlled trial. Journal of medical Internet research, 14(3).

Singh, K., Drouin, K., Newmark, L. P., Lee, J., Faxvaag, A., Rozenblum, R., ... \& Bates, D. W. (2016). Many mobile health apps target high-need, high-cost populations, but gaps remain. Health Affairs, 35(12), 2310-2318.

Sniehotta, F. F., Presseau, J., \& Araújo-Soares, V. (2014). Time to retire the theory of planned behaviour. Health Psychology Review, 8,1 -7. doi:10.1080/17437199.2013.869710

Spruijt-Metz, D., Hekler, E., Saranummi, N., Intille, S., Korhonen, I., Nilsen, W., Rivera, D. E., Spring, B., Michie, S., Asch, D. A., Sanna, A., Salcedo, V. T., Kukakfa, R., Pavel, M. (2015). Building new computational models to support health behavior change and maintenance: New opportunities in behavioral research. Translational Behavioral Medicine, 5(3), 335-346. doi:10.1007/s13142-015-0324-1

Stoyanov, S. R., Hides, L., Kavanagh, D. J., Zelenko, O., Tjondronegoro, D., \& Mani, M. (2015). Mobile App Rating Scale: A New Tool for Assessing the Quality of Health Mobile Apps. JMIR mHealth and uHealth, 3(1), e27. http://doi.org/10.2196/mhealth.3422

Stoyanov, S. R., Hides, L., Kavanagh, D. J., \& Wilson, H. (2016). Development and Validation of the User Version of the Mobile Application Rating Scale (uMARS). JMIR mHealth and uHealth, 4(2), e72. http://doi.org/10.2196/mhealth.5849

Strecher, V. J., McEvoy DeVellis, B., Becker, M. H., \& Rosenstock, I. M. (1986). The role of self-efficacy in achieving health behavior change, Health Education Quarterly, 13(1), 73-92.

Tatara, N., Årsand, E., Skrøvseth, S. O., \& Hartvigsen, G. (2013). Long-term engagement with a mobile self-management system for people with type 2 diabetes. JMIR mHealth and uHealth, 1(1).

Thomas, J. G., \& Bond, D. S. (2014). Review of innovations in digital health technology to promote weight control. Current Diabetes Reports, 14(5), 1-10. https://doi.org/10.1007/s11892-014-0485-1

Tuso, P. (2014). Prediabetes and lifestyle modification: Time to prevent a preventable disease. The Permanente Journal, 18(3), 88-93. https://doi.org/10.7812/TPP/14-002 Vervloet, M., Linn, A. J., van Weert, J. C., De Bakker, D. H., Bouvy, M. L., \& Van Dijk, L. (2012). The effectiveness of interventions using electronic reminders to improve 
adherence to chronic medication: a systematic review of the literature.Journal of the American Medical Informatics Association, 19(5), 696-704.

Wang, J. B., Ayala, G. X., Cadmus-Bertram, L. A., Cataldo, J. K., Madanat, H., Natarajan, L., Nichols, J., \& Pierce, J. P. White, M. M. (2016). Mobile and wearable device features that matter in promoting physical activity. Journal of Mobile Technology in Medicine, 5(2), 2-11.

Webb, T., Joseph, J., Yardley, L., \& Michie, S. (2010). Using the internet to promote health behavior change: a systematic review and meta-analysis of the impact of theoretical basis, use of behavior change techniques, and mode of delivery on efficacy. Journal of Medical Internet Research 12(1), 4. doi: 10.2196/jmir.1376

Yardley, L., Morrison, L., Bradbury, K., \& Muller, I. (2015). The person-based approach to intervention development: Application to digital health-related behavior change interventions. Journal of Medical Internet Research, 17(1), 30. doi: 10.2196/jmir.4055 